\title{
Investigation of Ultrasound as a Diagnostic Imaging Modality for Little League Shoulder
}

\author{
Shayne Fehr ${ }^{1}$ Gunnar Whealy ${ }^{1}$ Xue-Cheng Liu ${ }^{1}$ (1) \\ ${ }^{1}$ Department of Orthopaedic Surgery, Children's Wisconsin; Medical \\ College of Wisconsin, Milwaukee, Wisconsin, United States \\ Address for correspondence Xue-Cheng Liu, MD, PhD, Pediatric \\ Orthopaedics, 9000 W. Wisconsin Avenue, PO Box 1997, Suite 360, \\ Milwaukee, WI 53201, United States (e-mail: xcliu@mcw.edu).
} J Child Sci 2021;11:e240-e244.

\author{
Abstract

\section{Keywords} \\ - pediatric sports \\ medicine \\ - little league shoulder \\ - proximal physeal \\ epiphysiolysis \\ - musculoskeletal \\ ultrasound
}

Objective Ultrasound (US) is an established imaging modality in adult sports medicine but is not commonly used in the diagnosis of pediatric sports conditions, such as Little League shoulder (LLS). This study was conducted to determine the reliability of US measurement of width of the physis at the proximal humerus in diagnosed LLS and to compare US to radiography (RA) in detecting a difference between the affected (dominant) $(A)$ and unaffected $(U)$ shoulders.

Materials and Methods Ten male baseball players diagnosed with LLS were enrolled in the study. US images of the proximal humeral physis at the greater tuberosity of both shoulders were obtained by an US-trained sports medicine physician, and the physeal width was measured. Blinded to prior measurements, a separate physician performed measurements on the stored US images. Measurements were compared with RA on the anteroposterior (AP) view for both $\mathrm{A}$ and $\mathrm{U}$ at the time of the initial visit and for $\mathrm{A}$ at follow-up.

Results The physeal width $(\mathrm{mm})$ at $A$ and $U$ at the initial visit averaged $5.94 \pm 1.69$ and $4.36 \pm 1.20$ respectively on RA, and $4.15 \pm 1.12$ and $3.40 \pm 0.85$ on US. Median difference of averaged US measurements between $A$ and $U$ at initial evaluation was $0.75 \mathrm{~mm}(p=0.00016)$. A linear model showed US measurements to be predictive of $R A$ on $A\left(R^{2}=0.51\right)$ and $U\left(R^{2}=0.48\right)$.

Conclusion US was able to reliably measure the width of the proximal humeral physis and detect a difference between A and U. US correlated well with RA (standard for LLS). US should be considered by the US-trained physician for the diagnosis of LLS.

\section{Introduction}

Youth participation in organized sports continues to be popular in the United States. A recent report by the Aspen Institute identified a $73.2 \%$ participation rate in sports for children aged 6 to 12 years in 2019, with over 4 million regular participants in baseball. ${ }^{1}$ While participation in sport has clear benefits, a concerning reality in youth sports today is early sport specialization, which may be related to increasing injuries and burnout, ${ }^{2-4}$ especially for youth baseball

received

December 14, 2020

accepted after revision

July 25, 2021
DOI https://doi.org/ 10.1055/s-0041-1735535. ISSN 2474-5871. pitchers. Youth baseball is no exception. A prospective study by Fleisig et al found that $5 \%$ of youth baseball pitchers had arm surgery or retired from baseball due to arm injuries over a 10 -year period. ${ }^{5}$ Up to $70 \%$ of pitchers have reported arm pain in a season. ${ }^{6}$

One common cause of throwing-related pain is Little League shoulder (LLS), also known as proximal humeral epiphysiolysis, an overuse injury resulting most commonly from repetitive high-speed pitching in youth. ${ }^{7-9}$ With the increased availability and participation in competitive youth

\section{(C) 2021. The Author(s)}

This is an open access article published by Thieme under the terms of the Creative Commons Attribution License, permitting unrestricted use, distribution, and reproduction so long as the original work is properly cited. (https://creativecommons.org/licenses/by/4.0/) Georg Thieme Verlag KG, Rüdigerstraße 14, 70469 Stuttgart, Germany 
sports, the incidence of LLS is on the rise. ${ }^{10,11}$ It is most commonly seen in throwing athletes between 11 and 16 years of age. ${ }^{10}$ LLS commonly presents with pathology at the proximal humeral physeal plate ${ }^{12}$ and presents over a wide spectrum, from delayed physeal closure and physeal widening, to acute transphyseal fracture. ${ }^{13}$ Diagnosis of LLS is typically based on clinical evaluation and confirmation with radiography (RA). ${ }^{10}$ Chronic upper extremity overuse injuries such as LLS in competitive pediatric athletes yield imaging findings can be obvious and characteristic or subtle and atypical. ${ }^{11}$ Effective imaging tools are necessary for prompt and reliable interpretation that expedites management, returning the pediatric athlete to the playing field while minimizing long-term adverse outcomes. ${ }^{14}$

The use of musculoskeletal US has become common in the adult sports medicine clinic, and training is required in all sports medicine fellowships by the Accreditation Council for Graduate Medical Education. ${ }^{15}$ US is used for the diagnosis of soft tissue injuries and conditions and improves the accuracy of many injections. ${ }^{16,17}$ However, evidence is lacking regarding the use of US in pediatric sports injuries and conditions such as LLS, where RA and MRI are typically used. ${ }^{17}$ Evaluation with US can mitigate the radiation and costs associated with RA, as well as improve patient wait times when performed in clinic by the examining provider. ${ }^{18}$

The purpose of this study was to evaluate the effectiveness of in-clinic US in measuring the width of the lateral aspect of the proximal humeral physis. The goals of this study were threefold (1) to determine the reliability of US for the measurement of affected (A) or unaffected (U) physeal width; (2) to compare the differences of the physeal width of $A$ and $U$ between US and RA at the first clinic visit and 6week follow-up; and (3) to correlate measurements between US and RA using a linear model.

\section{Materials and Methods}

IRB approval was granted at Children's Wisconsin prior to the study. Informed consent and assent were obtained for each subject. Ten male baseball players with a new diagnosis of LLS based on clinical exam and RA findings were enrolled in the study at the time of their initial evaluation in clinic. The physeal width at the lateral aspect of the proximal humerus (greater tuberosity) was measured with RA by using picture archiving and communication system (PACS) on an anteroposterior (AP) view for both the $A$ and $U$ at the initial visit and $A$ at the follow-up visit (5-7 weeks later). Physeal width was calculated by using the measurement calipers, where the width was marked between the lateral corner of the proximal humeral metaphysis and the lateral corner of the epiphysis for both sides. Measurement was taken in $\mathrm{mm}$ (-Fig. 1A-C). After RA, a variable mid-range intensity linear US probe (GE NextGen Logic machine, Milwaukee, Wisconsin, United States) was used to obtain images of the shoulder sat the initial and follow-up visit using a long axis view relative to the humerus at the greater tuberosity (-Fig. 2). A similar arm position in the AP view of shoulder RA was required for US measurements. The US images were obtained
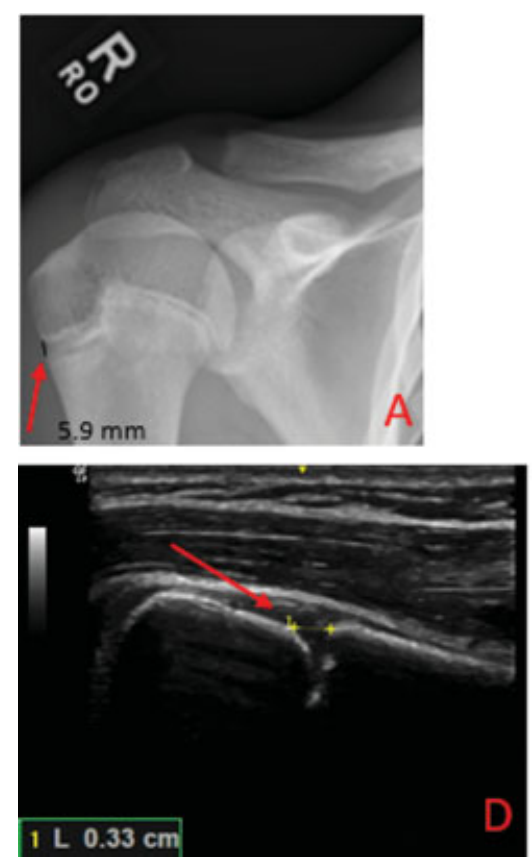
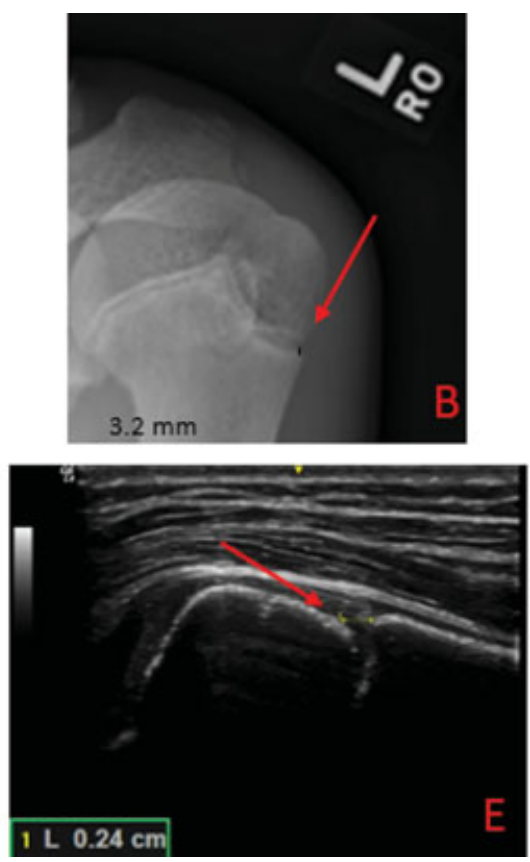

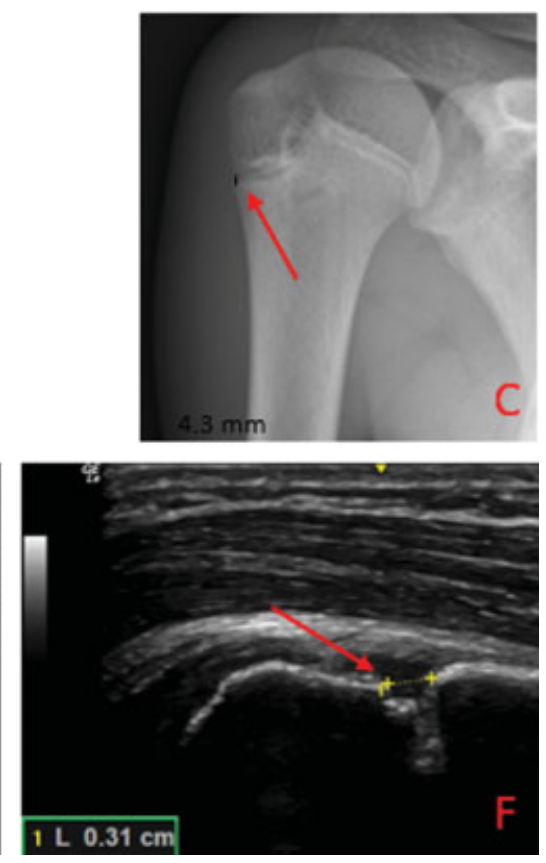

Fig. 1 (A) RA affected humerus, initial visit (red arrow points to physis: width was measured on the anteroposterior projection at the proximal humerus on picture archiving and communication system by using the measurement calipers, where the width was marked between the lateral corner of the proximal humeral metaphysis and the lateral corner of the epiphysis for both sides and was taken in mm). (B) RA unaffected humerus, initial visit (red arrow points to physis). (C) RA affected humerus, follow-up (red arrow points to physis). (D) US affected humerus, initial visit (red arrow points to physis: width was measured on the longitudinal US image at the greater tuberosity on the using the measurement calipers, where the width was marked between the hyperechoic corner of the proximal humeral metaphysis and the hyperechoic corner of the epiphysis and was taken in $\mathrm{cm}$ and converted to $\mathrm{mm}$ ). (E) US unaffected humerus, initial visit (red arrow points to physis). (F) US affected humerus, follow-up (red arrow points to physis). RA, radiography; US, ultrasound. 


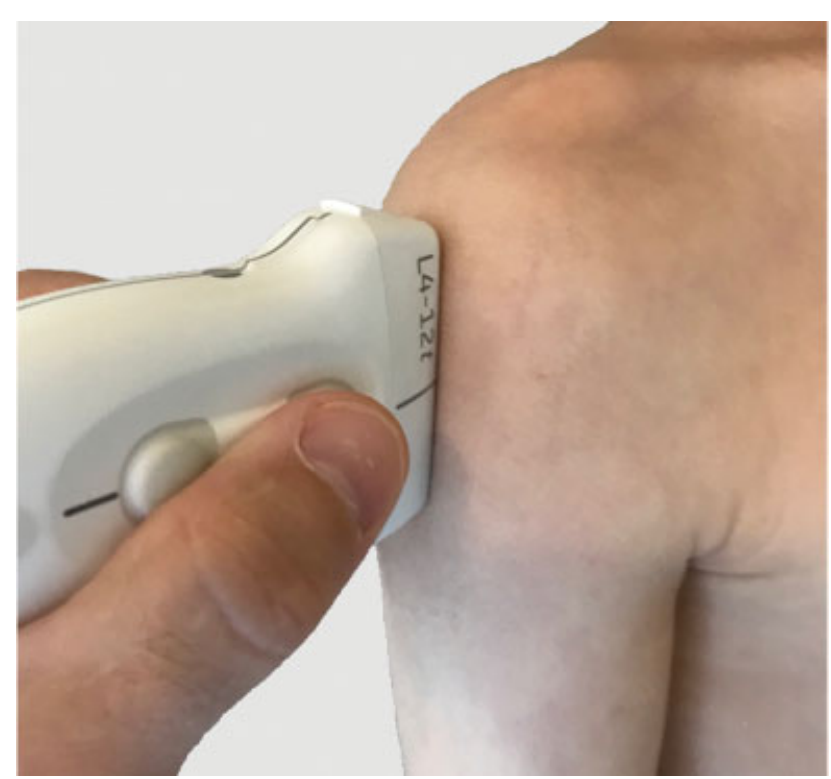

Fig. 2 Ultrasound probe placement. Probe is in longitudinal view relative to the humerus at the greater tuberosity.

by a US-trained pediatric sports medicine physician. The physeal width was measured on the longitudinal US image at the greater tuberosity on using the measurement calipers, where the width was marked between the hyperechoic corner of the proximal humeral metaphysis and the hyperechoic corner of the epiphysis. Measurement was taken in $\mathrm{cm}$ and converted to $\mathrm{mm}$ (-Fig. 1D-F). Blinded to prior measurements, a separate physician viewed US images and performed measurements of the physis. Both physicians measured each image twice.

Patients with a closed physis, history of fall, trauma, or deformity of the proximal humerus, and known genetic conditions related low bone density or bone fragility were excluded from this study. A pain scale from 0 to 10 (Wong-Baker FACES Pain Rating Scale) was used to assess the pain during the first visit and the follow-up visit.

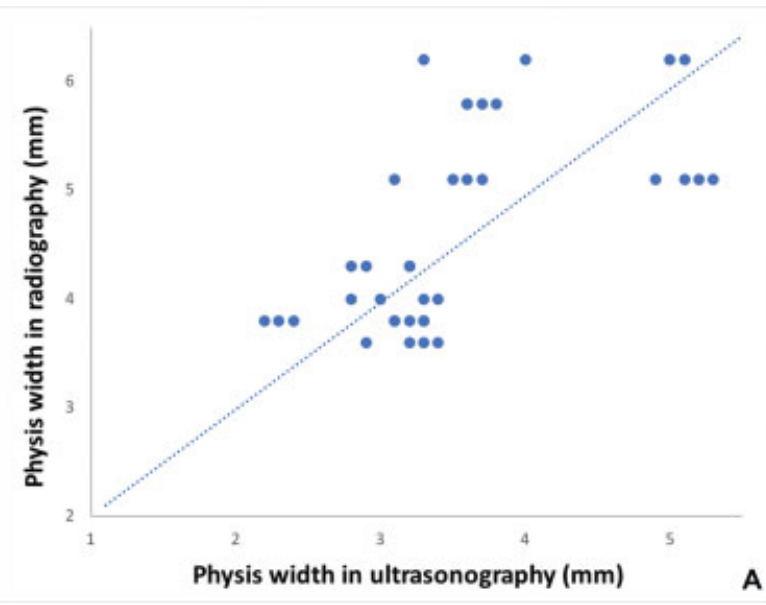

Statistical analysis was performed by using R 3.1.2 software (http://www.cran.r-project.org). A t-test was performed on US difference between A and U. Significance level was set at $p<0.05$. Linear regression models were used to evaluate trial effect (intrarater reliability) and inter-rater reliability. Intraclass correlation coefficients were calculated from the models. A linear model was used for prediction of RA measurement from US measurement.

\section{Results}

Ten male baseball players aged 12 to 16 years participated in this pilot study. Four subjects did not return appropriately in the follow-up timeframe and accordingly, and did not have follow-up measurements. High interrater reliability on US measurements was noted: A ICC $=0.9449$ (0.8667-0.9480) and U ICC $=0.8905(0.7503-0.9673)$. Both examiner 1 and examiner 2 have the same intrarater reliability on US measurements: A ICC $=0.9996(0.9984-0.9999)$ and U ICC $=$ 0.9999 (0.9996-1).

The physeal width $(\mathrm{mm})$ of $A$ and $U$ at the initial visit averaged $5.94 \pm 1.69$ and $4.36 \pm 1.20$ respectively on RA, and $4.15 \pm 1.12$ and $3.40 \pm 0.85$ on US. Median difference of all averaged US measurements between $A$ and $U$ at the initial evaluation was $0.75 \mathrm{~mm}(\mathrm{SE}=0.12)$, which was statistically significant $(p<0.001)$. This significance was also present when evaluating blinded and unblinded measurements separately ( $p=0.00013$ and $p=0.00020$, respectively). For the six patients who came for a follow-up appointment, the pain scale along with measurements were compared with see the changes between visits. Initial pain at rest and during activity was $1.5 \pm 1.38$ and $7 \pm 1.55$, respectively. All of the patients showed clinical improvement, and the follow-up pain at rest was 0 . The patients had not returned to throwing activity, so activity-related pain could not truly be assessed. The average reduction of the physis using RA was $1.43 \pm 1.30(\mathrm{~mm})$ and when using US was $0.62 \pm 0.49$ ( $\mathrm{mm}$ ).

A linear model showed US measurements to be predictive of RA measurements on $A$ (adjusted $R^{2}=0.51$; - Fig. 3A)

Fig. 3 (A) Linear model showing the relationship between US measurements and RA measurements of affected humerus (A) (adjusted $\mathrm{R}^{2}=0.51$ ). (B) Linear model showing the relationship between US measurements and RA measurements of unaffected humerus (U) (adjusted $\left.\mathrm{R}^{2}=0.48\right)$. RA, radiography; US, ultrasound. 
and $U$ (adjusted $R^{2}=0.48$; - Fig. $3 B$ ). A reduction of the physis width in both RA and US was reported from the initial visit to follow-up (-Fig. 1A-F).

\section{Discussion}

We were able to measure the physeal width of the proximal humerus reliably and were able to detect a difference between the affected (dominant) and unaffected shoulders. Our measurements also correlated well with RA measurements. In our study, reduction of pain on the affected shoulder at rest (1.5-0) coincided with decreases of physeal width in US ranging from a mean of 4.15 to $3.40 \mathrm{~mm}$. Future studies with larger sample sizes could evaluate this relationship further. We would hypothesize that athletes with continued pain at rest at follow-up would show lack of improvement or worsening of physeal width at follow-up due to nonadherence to the treatment plan.

US has been well established for musculoskeletal evaluations in the clinic. ${ }^{19}$ However, there is a lack of evidence for pediatric sports injuries and conditions, especially of the physis. A study by Lee et al showed US efficacy in the diagnosis of medial epicondyle lesions as compared with RA and MRI, with US providing a good PPV of medial epicondyle lesions. ${ }^{20}$ Through systematic literature review, Katzer et al concluded that there are hints of a comparable diagnostic accuracy of RA and US-based diagnosis of forearm fractures in children, in addition to being less painful, time saving, and cost-effective. ${ }^{18}$

Comparing these pediatric applications with others as well as our results, the use of US for diagnosis in the pediatric sports patient appears promising. To the patient, US is fundamentally safer than RA, as no radiation is involved. ${ }^{21}$ Also, using an in-clinic model, it is likely less expensive than RA with lower equipment costs and overhead. ${ }^{16,22}$ Efficiency is also likely improved compared with RA, as the patient does not have leave the clinic room for RA and the read is done immediately by the physician. ${ }^{17}$ It is important to note that a limited evaluation of the lateral shoulder with US will not identify other possible injuries or conditions of the soft tissues seen on a complete diagnostic exam. Also, the known limitations of US to evaluate bone and joint conditions should be considered to avoid missing diagnoses such as bone neoplasms, osteomyelitis, and joint derangements. Furthermore, US is very operator-dependent ${ }^{23}$ and this may impact the acquisition and interpretation of images. Acquiring US images is a skill that requires training and practice, such as completion of an ACGME-accredited sports medicine fellowship. ${ }^{15}$ However, experienced sports medicine physicians should be able to reliably image and measure the physis to confirm the presence of widening in the patient with clinical high suspicion of LLS (-Fig. 1A-F).

We would like to acknowledge several limitations of this study. This is a small pilot study, and greater numbers could improve the strength of the findings and identity causes of bias. Further age-matched and controlled subject studies should be completed to detect minimal changes with accepted errors. Although detectable changes of US- measured physeal width averaged $0.75 \mathrm{~mm}(0.05-1.3 \mathrm{~mm})$ between affected and contralateral shoulder and $0.62 \mathrm{~mm}$ $(0.05-1.2 \mathrm{~mm})$ on the injured shoulder between two visits, variation in the appearance in the physis makes accurate measurements difficult. However, US is able to properly identify the distances between bone structures in the neonatal clubfoot. ${ }^{24}$ The thickness of the soft tissue that measurements were being taken through was $2.1 \pm 0.6$ $(\mathrm{mm})$ for clubfoot and $0.5 \pm 0.4(\mathrm{~mm})$ for normal. Additionally, US was able to take measurements of cuboid-calcaneus distance ranging from $1.0 \pm 1.1$ to $2.5 \pm 1.3(\mathrm{~mm}),{ }^{24}$ which fell into the range of our US measurements as well as minimally detectable changes for the cartilage. They also showed that there were very high specificity and sensitivity of the US to dealing with rotator cuff tendon tears, ranging from 0.86 to 0.97 for specificity and from 0.8 to 0.96 for sensitivity. ${ }^{25}$ Regarding measurement bias, we attempted to minimize this by having a blinded separate physician to evaluate the US images independently, also without visualization of RA images for each patient. Each image was viewed at the same sitting with its comparison image, so it would have been assumed that a difference may exist. To eliminate bias further, we could have completely de-identified and randomized the images. However, comparing the affected shoulder with its paired unaffected shoulder is consistent with real-time clinical practice.

\section{Conclusion}

US should be considered as an alternate modality for the UStrained sports medicine physician for evaluating physeal widening of the proximal humeral epiphysis in the diagnosis of LLS. However, RA should still be considered the imaging modality of choice in clinical scenarios that are less certain based on history and exam to avoid the risk of not recognizing a clinically significant condition not identified by this limited approach.

\section{Funding \\ None. \\ Conflict of Interest \\ None declared.}

\section{References}

1 State of Play 2020: Pre-Pandemic Trends Ages 6-12. The Aspen Institute Project Play. Accessed January 25, 2021 at: https://www. aspenprojectplay.org/state-of-play-2020/ages-6-12

2 DiFioriJP, Benjamin HJ, Brenner JS, et al. Overuse injuries and burnout in youth sports: a position statement from the American Medical Society for Sports Medicine. Br J Sports Med 2014;48(04):287-288

3 Myer GD, Jayanthi N, Difiori JP, et al. Sport specialization, part I: does early sports specialization increase negative outcomes and reduce the opportunity for success in young athletes? Sports Health 2015;7(05):437-442

4 Popkin CA, Bayomy AF, Ahmad CS. Early sport specialization. J Am Acad Orthop Surg 2019;27(22):e995-e1000

5 Fleisig GS, Andrews JR, Cutter GR, et al. Risk of serious injury for young baseball pitchers: a 10-year prospective study. Am J Sports Med 2011;39(02):253-257 
6 Makhni EC, Morrow ZS, Luchetti TJ, et al. Arm pain in youth baseball players: a survey of healthy players. Am J Sports Med 2015;43(01):41-46

7 Adams JE. Little league shoulder: osteochondrosis of the proximal humeral epiphysis in boy baseball pitchers. Calif Med 1966;105 (01):22-25

8 Carson WG Jr, Gasser SI. Little Leaguer's shoulder. A report of 23 cases. Am J Sports Med 1998;26(04):575-580

9 Sabick MB, Kim YK, Torry MR, Keirns MA, Hawkins RJ. Biomechanics of the shoulder in youth baseball pitchers: implications for the development of proximal humeral epiphysiolysis and humeral retrotorsion. Am J Sports Med 2005;33(11):1716-1722

10 Osbahr DC, Kim HJ, Dugas JR. Little league shoulder. Curr Opin Pediatr 2010;22(01):35-40

11 Heyworth BE, Kramer DE, Martin DJ, Micheli LJ, Kocher MS, Bae DS. Trends in the presentation, management, and outcomes of little league shoulder. Am J Sports Med 2016;44(06):1431-1438

12 Murachovsky J, Ikemoto RY, Nascimento LG, Serpone Bueno R, Strose E, Almeida LH. Does the presence of proximal humerus growth plate changes in young baseball pitchers happen only in symptomatic athletes? An $\mathrm{x}$ ray evaluation of 21 young baseball pitchers. Br J Sports Med 2010;44(02):90-94

13 Saltzman BM, Chalmers PN, Mascarenhas R, Cole BJ, Romeo AA. Upper extremity physeal injury in young baseball pitchers. Phys Sportsmed 2014;42(03):100-111

14 Paz DA, Chang GH, Yetto JM Jr, Dwek JR, Chung CB. Upper extremity overuse injuries in pediatric athletes: clinical presentation, imaging findings, and treatment. Clin Imaging 2015;39 (06):954-964

15 ACGME Program Requirements for Graduate Medical Educationin Sports Medicine. Accessed January 25, 2021 at: https://www. acgme.org/Portals/0/PFAssets/ProgramRequirements/116_SportsMedicine_2020.pdf?ver=2020-02-25-151514-123
16 Nazarian LN. The top 10 reasons musculoskeletal sonography is an important complementary or alternative technique to MRI. AJR Am J Roentgenol 2008;190(06):1621-1626

17 Piccolo CL, Galluzzo M, Ianniello S, et al. Pediatric musculoskeletal injuries: role of ultrasound and magnetic resonance imaging. Musculoskelet Surg 2017;101(Suppl 1):85-102

18 Katzer C, Wasem J, Eckert K, Ackermann O, Buchberger B. Ultrasound in the diagnostics of metaphyseal forearm fractures in children: a systematic review and cost calculation. Pediatr Emerg Care 2016;32(06):401-407

19 DiPietro MA, Leschied JR. Pediatric musculoskeletal ultrasound. Pediatr Radiol 2017;47(09):1144-1154

20 Lee YY, Yang TH, Huang CC, et al. Ultrasonography has high positive predictive value for medial epicondyle lesions among adolescent baseball players. Knee Surg Sports Traumatol Arthrosc 2019;27(10):3261-3268

21 Lin S. Procedural ultrasound in pediatric patients: techniques and tips for accuracy and safety. Pediatr Emerg Med Pract 2016;13 (06):1-38, quiz 24-25

22 Weide G, van der Zwaard S, Huijing PA, Jaspers RT, Harlaar J. 3D ultrasound imaging: fast and cost-effective morphometry of musculoskeletal tissue. J Vis Exp 2017;(129):

23 Chang RF, Lee CC, Lo CM. Computer-aided diagnosis of different rotator cuff lesions using shoulder musculoskeletal ultrasound. Ultrasound Med Biol 2016;42(09):2315-2322

24 Aurell Y, Johansson A, Hansson G, Wallander H, Jonsson K. Ultrasound anatomy in the normal neonatal and infant foot: an anatomic introduction to ultrasound assessment of foot deformities. Eur Radiol 2002;12(09):2306-2312

25 Henderson REA, Walker BF, Young KJ. The accuracy of diagnostic ultrasound imaging for musculoskeletal soft tissue pathology of the extremities: a comprehensive review of the literature. Chiropr Man Therap 2015;23:31 